\title{
A HYPERBOLIC COUNTERPART TO ROKHLIN'S COBORDISM THEOREM
}

\author{
MICHELLE CHU AND ALEXANDER KOLPAKOV
}

\begin{abstract}
The purpose of the present paper is to prove existence of super-exponentially many compact orientable hyperbolic arithmetic $n$-manifolds that are geometric boundaries of compact orientable hyperbolic $(n+1)$-manifolds, for any $n \geq 2$, thereby establishing that these classes of manifolds have the same growth rate with respect to volume as all compact orientable hyperbolic arithmetic $n$-manifolds. An analogous result holds for non-compact orientable hyperbolic arithmetic $n$-manifolds of finite volume that are geometric boundaries, for $n \geq 2$.
\end{abstract}

In homage to $V$. Rokhlin on his 100th anniversary.

\section{INTRODUCTION}

A classical result by V. Rokhlin states that every compact orientable 3-manifold bounds a compact orientable 4-manifold, and thus the three-dimensional cobordism group is trivial. Rokhlin also proved that a compact orientable 4-manifold bounds a compact orientable 5-manifold if and only if its signature is zero, which is true for all closed orientable hyperbolic 4-manifolds. One can recast the question of bounding in the setting of hyperbolic geometry, which generated plenty of research directions over the past decades.

A hyperbolic manifold is a manifold endowed with a Riemannian metric of constant sectional curvature -1 . Throughout the paper, hyperbolic manifolds are assumed to be connected, orientable, complete, and of finite volume, unless otherwise stated. We refer to [34, 45] for the definition of an arithmetic hyperbolic manifold.

A connected hyperbolic $n$-manifold $\mathcal{M}$ is said to bound geometrically if it is isometric to $\partial \mathcal{W}$ for a hyperbolic $(n+1)$-manifold $\mathcal{W}$ with totally geodesic boundary.

Indeed, some interest in hyperbolic manifolds that bound geometrically was kindled by the works of Long, Reid [28, 29] and Niemershiem [36], motivated by a preceding work of Gromov [15, 16] and a question by Farrell and Zdravkovska [13]. This question is also related to hyperbolic instantons, as described in [40, 41].

As [28] shows many closed hyperbolic 3-manifolds do not bound geometrically: a necessary condition is that the $\eta$-invariant of the 3 -manifold must be an integer. The first example of a closed hyperbolic 3-manifold known to bound geometrically was constructed by Ratcliffe and Tschantz in [40] and has volume of order 200.

The first examples of knot and link complements that bound geometrically were produced by Slavich in 38, 39. However, 25] implies that there are plenty of cusped hyperbolic 3-manifolds that cannot bound geometrically, with the obstruction being the geometry of their cusps.

In [30, by using arithmetic techniques, Long and Reid built infinitely many orientable hyperbolic $n$-manifolds $\mathcal{N}$ that bound geometrically an $(n+1)$-manifold $\mathcal{M}$, in every dimension $n \geqslant 2$. Every such manifold $\mathcal{N}$ is obtained as a cover of some $n$-orbifold $O_{\mathcal{N}}$ geodesically immersed in a suitable $(n+1)$-orbifold $O_{\mathcal{M}}$. However, this construction gives no control on the volume of the manifolds.

M.C. was supported by NSF grant DMS-1803094 and A.K. was supported by SNSF project no. PP00P2-170560. 
In [4, Belolipetsky, Gelander, Lubotzky, and Shalev showed that the growth rate of all orientable arithmetic hyperbolic manifolds, up to isometry, with respect to volume is super-exponential, in all dimensions $n \geq 2$. Their lower bound used a subgroup counting technique due to Lubotzky [32]. In the present paper we shall use the ideas of [30] together with the subgroup counting argument due to Lubotzky [32] (also used in [4]), together with the more combinatorial colouring techniques from [27] in order to prove the following facts:

Proposition 1.1. Let $\kappa_{n}(x)=$ the number of non-isometric non-orientable compact arithmetic hyperbolic $n$-manifolds of volume $\leq x$. Then we have that $\kappa_{n}(x) \asymp x^{x}$ for any $n \geq 3$.

Proposition 1.2. Let $\nu_{n}(x)=$ the number of non-isometric non-orientable cusped arithmetic hyperbolic $n$-manifolds of volume $\leq x$. Then we have that $\nu_{n}(x) \asymp x^{x}$ for any $n \geq 3$.

Above, the notation " $f(x) \asymp x^{x}$ " for a function $f(x)$ is a shorthand for "there exist positive constants $A_{1}, B_{1}, A_{2}, B_{2}$, and $x_{0}$, such that $A_{1} x^{B_{1} x} \leq f(x) \leq A_{2} x^{B_{2} x}$, for all $x \geq x_{0} . "$

The techniques of 4, 32 provide us with super-exponentially many manifolds of volume $\leq x$ (for $x$ sufficiently large) by employing a retraction of the manifold's fundamental group into a free group. In our case we need however to take extra care in order to arrange for the kernel of such retraction comprise an orientation-reversing element. Here Coxeter polytopes and reflection groups come into play as natural sources of orientation-reversing isometries, as well as building blocks for manifolds.

Then, by using the embedding technique from [24] and the techniques for constructing torsionfree subgroups from [30] (see Lemma 3.1, also Lemma 3.2 below), we obtain the following theorems establishing that the growth rate with respect to volume of arithmetic hyperbolic manifolds bounding geometrically is the same as that over all arithmetic hyperbolic manifolds.

Theorem 1.3. Let $\beta_{n}(x)=$ the number of non-isometric orientable compact arithmetic hyperbolic $n$-manifolds of volume $\leq x$ that bound geometrically. Then we have that $\beta_{n}(x) \asymp x^{x}$ for $n \geq 3$.

Theorem 1.4. Let $\gamma_{n}(x)=$ the number of non-isometric orientable cusped arithmetic hyperbolic $n$-manifolds of volume $\leq x$ that bound geometrically. Then we have that $\gamma_{n}(x) \asymp x^{x}$ for $n \geq 3$.

As a by-product, we provide a different proof to a part of the results in [26] and construct a few new Coxeter polytopes not otherwise available on the literature. For dimensions $n=2, \ldots, 6$ in the compact case, and dimensions $n=2, \ldots, 13$ in the cusped case, we construct explicit examples of retractions onto free groups. More involved computations may be performed in dimensions $n=14,15$ (using the polytopes from [1]) and $n=18,19$ (using the polytopes from [21]). However, the general case follows from the main result of Bergeron, Haglund, Wise [5] on virtually retractions of arithmetic groups of simplest type onto geometrically finite subgroups.

It is also worth mentioning that a linear lower bound with respect to volume for the number of isometry classes of compact orientable bounding hyperbolic 3-manifolds was obtained previously in [33] by extending the techniques from [23] and comparing the Betti numbers of the resulting manifolds.

Given the present question's background, one may think of Theorem 1.3 as a "hyperbolic counterpart" to Rokhlin's theorem. Indeed, not every compact orientable arithmetic hyperbolic 3-manifold bounds geometrically, but the number of those that do has the same growth rate as the number of all compact orientable arithmetic hyperbolic 3-manifolds. In the light of Wang's theorem [46] and the results of [9], an analogous statement can be formulated for geometrically bounding hyperbolic 4-manifolds without arithmeticity assumption.

As for the closed hyperbolic surfaces that bound geometrically, it follows from the work of Brooks [6] that for each genus $g \geq 2$ the ones that bound form a dense subset of the Teichmüller space. Thus, there are infinitely many of them in each genus $g \geq 2$. However, there are only finitely many arithmetic ones, by [4. The argument of Theorem 1.3 applies in this case, and we obtain 
Theorem 1.5. Let $\alpha(g)=$ the number of non-isometric orientable closed arithmetic surfaces of genus $\leq g$ that bound geometrically. Then $(c g)^{\frac{g}{8}} \leq \alpha(g) \leq(d g)^{2 g}$, for some constants $0<c \leq d$.

Remark 1.6. An analogous statement holds for finite-area non-compact surfaces if we substitute the genus $g$ with the area $x$. Namely, then $(c x)^{\frac{x}{32 \pi}} \leq \alpha(x) \leq(d x)^{\frac{x}{2 \pi}}$, for some $0<c \leq d$.

This adds many more (albeit not very explicit) examples to the ones obtained by Zimmermann in [47, 48].

The manifolds that we construct in abundance in order to prove Theorem 1.3 - Theorem 1.5 all happen to be orientation double covers. An easy observation implies that any closed orientable manifold $M$ that is an orientation cover bounds topologically: consider $W^{\prime}=M \times[0,1]$ and quotient one of its boundary components by an orientation-reversing fixed point free involution that $M$ necessarily has in this case. The resulting manifold $W$ is orientable with boundary $\partial W \cong M$. Indeed, these are the manifolds that are not orientation covers that may make the cobordism group non-trivial.

Concerning geometrically bounding manifolds, we are not aware at the moment of any that does bound geometrically and that is not an orientation cover, in both compact and finite-volume cases.

\section{Constructing geodesic Boundaries by COlOuRings}

2.1. The right-angled dodecahedron. Let $\mathcal{D} \subset \mathbb{H}^{3}$ be a right-angled dodecahedron. By Andreev's theorem [2], it is realisable as a regular compact hyperbolic polyhedron. Suppose that the faces of $\mathcal{D}$ are labelled with the numbers $1, \ldots, 12$ as shown in Figure 1. Let $s_{i}$ be the reflection in the supporting hyperplane of the $i$-th facet of $\mathcal{D}$, for $i=1, \ldots, 12$, and let $\Gamma_{12}=\operatorname{Ref}(\mathcal{D})=$ $\left\langle s_{1}, s_{2}, \ldots, s_{12}\right\rangle$ be the corresponding reflection group.

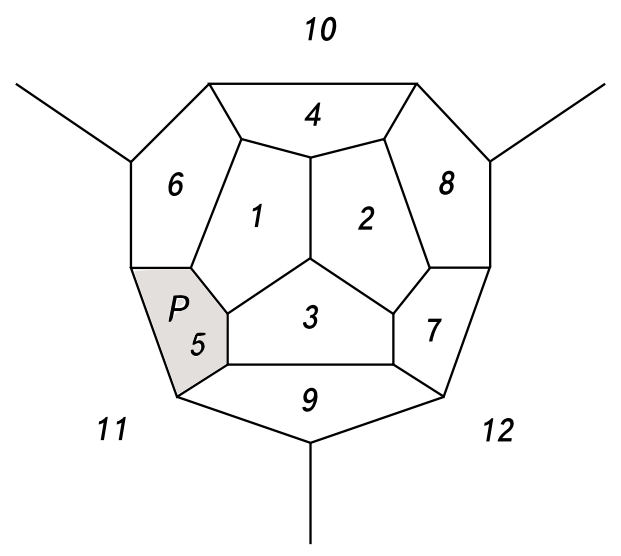

Figure 1. A face labelling for the dodecahedron $\mathcal{D}$.

Let $P$ be the pentagonal two-dimensional face of $\mathcal{D}$ labelled 5 and let $\Gamma_{4}=\left\langle s_{1}, s_{3}, s_{9}, s_{11}\right\rangle$ be an infinite-index subgroup of $\Gamma_{12}$, which we may consider as a reflection group acting on the supporting hyperplane of $P$, which is isometric to $\mathbb{H}^{2}$. There is a retraction $R$ of $\Gamma_{12}$ onto $\Gamma_{4}$ given by

$$
R: s_{i} \mapsto \begin{cases}s_{i}, & \text { if } i \in\{1,3,9,11\} \\ \text { id, } & \text { otherwise. }\end{cases}
$$

The group $\Gamma_{4}$ is virtually free: it contains $F_{3} \cong\langle x, y, z\rangle$, a free group of rank 3 , as an index 8 normal subgroup. Indeed, with $x=s_{1} s_{11}, y=\left(s_{1} s_{9}\right)^{2}, z=s_{1} s_{3} s_{11} s_{3}$, we have $F_{3}$ realised as a subgroup of $\Gamma_{4}$, which is the fundamental group of a 2 -sphere with four disjoint closed discs removed, as depicted in Figure 2. 
Let $P$ be a simple $n$-dimensional polytope (not necessarily hyperbolic) with $m$ facets labelled by distinct elements of $\Omega=\{1,2, \ldots, m\}$. A colouring of $P$, according to [12, 14, 20, 42, 43, is a map $\lambda: \Omega \rightarrow \mathbb{Z}_{2}^{n}$. A colouring is called proper if the colours of facets around each vertex of $P$ are linearly independent vectors of $V=\mathbb{Z}_{2}^{n}$.

Proper colourings of compact right-angled polytopes $P \subset \mathbb{H}^{n}$ give rise to interesting families of hyperbolic manifolds [14, 23, 42, 43]. Such polytopes $P$ are necessarily simple.

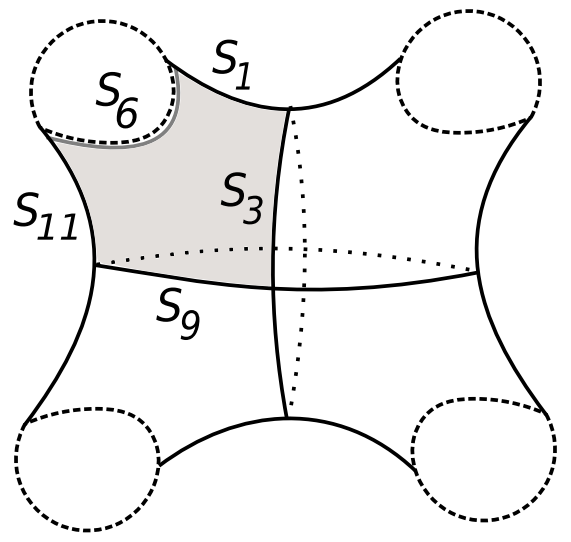

Figure 2. The sphere $\mathbb{S}^{2}$ with four disjoint closed discs removed, and one of eight tiles associated to $\Gamma_{4}$ shaded. The reflection side of $s_{6}$ completes this tile to the pentagon $P$, and removing it is equivalent to cutting out a closed disc.

In 23] the notion of a colouring is extended to let $V=\mathbb{Z}_{2}^{s}, s \geq 2$, be a finite-dimensional vector space over $\mathbb{Z}_{2}$, and in [27] the notion of colouring is extended to polytopes that are not necessarily simple, but rather satisfy a milder constraint of being simple at edges.

A polytope $P \subset \mathbb{H}^{n}$ is called simple at edges if each edge belongs to exactly $(n-1)$ facets. In the case of a finite-volume right-angled polytope $P \subset \mathbb{H}^{n}, P$ is simple if $P$ is compact, and $P$ is simple at edges if it has any ideal vertices.

A colouring of a polytope $P \subset \mathbb{H}^{n}$ which is simple at edges is a map $\lambda: \Omega \rightarrow V$, where $V=\mathbb{Z}_{2}^{s}$, $s \geq n$, is a finite-dimensional vector space over $\mathbb{Z}_{2}$. A colouring $\lambda$ is proper if the following two conditions are satisfied:

(1) Properness at vertices: if $v$ is a simple vertex of $P$, then the $n$ colours of facets around it are linearly independent vectors of $V$;

(2) Properness at edges: if $e$ is an edge of $P$, then the $(n-1)$ colours of facets around $e$ are linearly independent.

Given a fixed labelling $\Omega$ of the facets of a finite-volume right-angled polytope $P \subset \mathbb{H}^{n}$, we shall write its colouring as a vector $\lambda=\left(\lambda_{1}, \ldots, \lambda_{m}\right)$, where $\lambda_{i}=\sum_{k=0}^{\operatorname{dim} V-1} \lambda(i)_{k} \cdot 2^{k}$ is a binary representation of the vector $\lambda(i) \in V$, for all $i \in \Omega$.

Let $s_{i}$ be a reflection in the supporting hyperplane of the $i$-th facets of $P$. Then a proper colouring $\lambda: \Omega \rightarrow V$ defines a homomorphism from the reflection group $\Gamma=\operatorname{Ref}(P)=\left\langle s_{1}, s_{2}, \ldots, s_{m}\right\rangle$ of $P$ to $V$, such that ker $\lambda$ is a torsion-free subgroup of $\Gamma$ [27].

Let us consider one of the colourings of $\mathcal{D}$ defined in [14, Table 1], that gives rise to a nonorientable manifold cover of the orbifold $\mathbb{H}^{3} / \Gamma_{12}$. Namely, choose $\lambda=(1,2,4,4,2,6,3,5,5,3,1,7)$, so that the $i$-th component of $\lambda$ corresponds to the colour $\lambda_{i}$ of the $i$-th face of $\mathcal{D}$. As follows from [23, Corollary 2.5], this colouring is indeed non-orientable, since $\lambda_{1}+\lambda_{2}+\lambda_{7}=\mathbf{0}$ in $\mathbb{Z}_{2}^{3}$. Thus, $M=\mathbb{H}^{3} / \Gamma$, with $\Gamma=\operatorname{ker} \lambda$ a torsion-free subgroup of $\Gamma_{12}$, is a non-orientable compact hyperbolic 3-manifold. 
The reflection group $\Gamma_{12}$ is an index 120 subgroup in the reflection group $\operatorname{Ref}(T)$ of the orthoscheme $T=[4,3,5]$, which is arithmetic. Thus, $\Gamma_{12}$ is also arithmetic. Moreover, $\operatorname{Ref}(T)=$ $O^{+}(q, \mathbb{Z}[\omega])$, with $\omega=\frac{1+\sqrt{5}}{2}$, for the quadratic form $q=-\omega x_{0}^{2}+x_{1}^{2}+x_{2}^{2}+x_{3}^{2}$, as described in [3, $\S 7]$ and, initially, in [7].

Next, let $\rho: \Gamma \rightarrow R(\Gamma)$ be the restriction of $R$. Observe that $R(\Gamma)=\Gamma_{4}$, and thus $\rho: \Gamma \rightarrow \Gamma_{4}$ is an epimorphism. Here we use the fact that $s_{1}=\rho\left(s_{1} s_{2} s_{7}\right), s_{3}=\rho\left(s_{3} s_{4}\right), s_{9}=\rho\left(s_{8} s_{9}\right)$, and $s_{11}=\rho\left(s_{2} s_{10} s_{11}\right)$, where all the respective products of $s_{i}$ 's belong to $\Gamma=\operatorname{ker} \lambda$.

For any subgroup $K \leq F_{3}$ of index $n$, let us consider $\rho^{-1}(K)=R^{-1}(K) \cap \Gamma$. Then $K$ has index $8 n$ in $\Gamma_{4}$, and $H=\rho^{-1}(K)$ has index $8 n$ in $\Gamma$.

Moreover, we produce an orientation-reversing element $\delta \in \Gamma$, such that $\delta \in H$ for every such $H$.

Having established these facts, we know that there are $\asymp n^{n}$ non-conjugate in $\operatorname{Isom}\left(\mathbb{H}^{3}\right)$ subgroups of $\Gamma$ by using the argument of [4, $\S 5.2]$, and thus there are $\asymp x^{x}$ non-isometric non-orientable compact arithmetic 3-manifolds $M=\mathbb{H}^{3} / H$ of volume $\leq x$ (for $x>0$ big enough). This proves the three-dimensional case of Proposition 1.1.

Now, observe that $x=s_{1} s_{11}$, and $\lambda(x)=(1,0,0)^{t}+(1,0,0)^{t}=\mathbf{0}$ in $\mathbb{Z}_{2}^{3}$. Similarly, $\lambda(y)=\lambda(z)=$ 0. Also, $R$ maps $x, y$, and $z$ respectively to themselves. Thus, $F_{3}=\langle x, y, z\rangle \subset \rho(\Gamma)$. Finally, the element $\delta=s_{2} s_{4} s_{6}$ is such that $\lambda(\delta)=(0,1,0)^{t}+(1,0,0)^{t}+(1,1,0)^{t}=\mathbf{0}$, and $\rho(\delta)=\mathrm{id}$, so that $\delta \in \Gamma$ and $\delta \in H=\rho^{-1}(K)$, for every $K \leq F_{3}$.

Given that $H \leq O^{+}(q, \mathbb{Z}[\omega])$ for an admissible quadratic form $q$, we have that the argument in the proof of [24, Corollary 1.5] applies in this case, and thus the non-orientable compact manifold $M=\mathbb{H}^{3} / H$ embeds into a compact orientable manifold $N=\mathbb{H}^{4} / G$, for some arithmetic torsionfree $G \leq O^{+}(Q, \mathbb{Z}[\tau])$, with $Q=q+x_{4}^{2}$. Then, cutting $N$ along $M$ produces a manifold $N / / M$, which is connected since $N$ is orientable while $M$ is not. Also, since $M$ is a one-sided submanifold of $N$, the boundary $\partial N^{\prime}$ is isometric to $\widetilde{M}$, the orientation cover of $M$. Thus we obtain a collection of $\asymp n^{n}$ orientable arithmetic 3 -manifolds $\widetilde{M}$ that bound geometrically. However, some of them can be isometric, since the same manifold $\widetilde{M}$ can be the orientation cover of several distinct non-orientable manifolds $N_{1}, \ldots, N_{m}$.

In order to estimate $m$, observe that each $N_{i}$ is a quotient of $\widetilde{M}$ by a fixed point free orientationreversing involution. Let the number of such involutions for $\widetilde{M}$ be $I(\widetilde{M})$. Then $m \leq I(\widetilde{M}) \leq$ $|\operatorname{Isom}(\widetilde{M})| \leq c_{1} \cdot \operatorname{Vol}(\widetilde{M}) \leq c_{2} \cdot n=c_{3} x$. Indeed, the isometry group of $\widetilde{M}$ is finite, and by the Kazhdan-Margulis theorem [22] there exists a lower bound for the volume of the orbifold $\widetilde{M} / \operatorname{Isom}(\widetilde{M}) \geq c_{0}>0$, from which the final estimate follows. Thus, we have at least $\asymp n^{n} /\left(c_{2} n\right) \asymp$ $n^{n} \asymp x^{x}$ non-isometric compact orientable arithmetic hyperbolic 3 -manifolds $\widetilde{M}$ of volume $\leq x$ that bound geometrically. The upper-bound of the same order of growth follows from [4. This proves the three-dimensional case of Theorem 1.3.

2.2. The right-angled 120-cell. Let $\mathcal{C} \subset \mathbb{H}^{4}$ be the regular right-angled 120-cell. This polytope can be obtained by the Wythoff construction with the orthoscheme $[4,3,3,5]$ that uses the vertex stabiliser subgroup $[3,3,5]$ of order $(120)^{2}=14400$. The polytope $\mathcal{C}$ is compact and each of its 3 -dimensional facets is a regular right-angled dodecahedron isometric to $\mathcal{D}$ defined above.

Let us choose a facet $F$ of $\mathcal{C}$ and label it 120. Since $F$ is isometric to $\mathcal{D}$, we can label the neighbouring facets of $F$ as follows:

- choose an isometry $\varphi$ between $F$ and $\mathcal{D}$ and transfer the labelling of 2-dimensional faces of $\mathcal{D}$ depicted in Figure 1 from $\mathcal{D}$ to $F$ via $\varphi$,

- if $F^{\prime}$, a facet of $\mathcal{C}$, shares a 2-face labelled $i \in\{1,2, \ldots, 12\}$ with $F$, label $F^{\prime}$ with $i$.

The remaining facets of $\mathcal{C}$ can be labelled with the numbers in $\{13, \ldots, 119\}$ in an arbitrary way. Let $s_{i}$ denote the reflection on the supporting hyperplane of the $i$-th facet of $\mathcal{C}$, and let $\Gamma_{120}=$ $\operatorname{Ref}(\mathcal{C})=\left\langle s_{1}, s_{2}, \ldots, s_{120}\right\rangle$. 


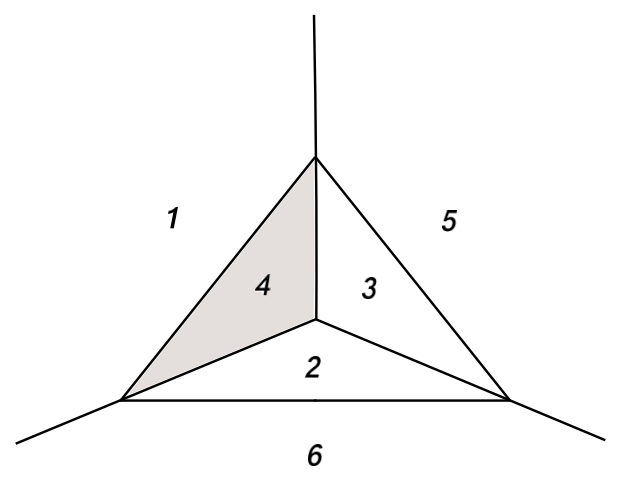

Figure 3. A face labelling for the bi-pyramid $\mathcal{R}_{3}$. The compact vertices in $\mathbb{H}^{3}$ are the central one and the one at $\infty$. All other vertices are ideal and belong to $\partial \mathbb{H}^{3}$.

Now define a colouring $\Lambda$ of $\mathcal{C}$ by using the colouring $\lambda$ of $\mathcal{D}$ defined above. Namely, we set

$$
\Lambda\left(s_{i}\right)=\left\{\begin{array}{cl}
\lambda_{i}, & \text { for } 1 \leq i \leq 12, \\
2^{i-10}, & \text { for } 13 \leq i \leq 120 .
\end{array}\right.
$$

Observe that $\Lambda$ is a proper colouring of $\mathcal{C}$, as defined in [23], and thus $\Gamma=\operatorname{ker} \Lambda$ is torsion-free. Also, $\Lambda$ is a non-orientable colouring. As in the case of $\mathcal{D}$, we use the retraction $R$ in order to map $\Gamma_{120}$ onto $\Gamma_{4}$, that contains $F_{3}$ as a finite-index subgroup. By taking preimages $H=\rho^{-1}(K)$ in $\Gamma$ of index $n$ subgroups $K \leq F_{3}$ and applying our argument from the previous section, we complete the proof of Proposition 1.1 in the 4-dimensional case and obtain $\asymp n^{n}$ non-isometric non-orientable compact arithmetic hyperbolic 4-manifolds $M=\mathbb{H}^{4} / H$. The rest of the argument follows from [24, Theorem 1.4]. Thus, the 4-dimensional case of Theorem 1.3 is also proven.

2.3. Non-compact right-angled polytopes. Let $\mathcal{R}_{3}$ be a right-angled bi-pyramid depicted in Figure 3, which is the first polytope in the series described by L. Potyagaulo and È. Vinberg in [37. The construction in [37] produces a series of polytopes $\mathcal{R}_{n} \subset \mathbb{H}^{n}$, for $n=3, \ldots, 8$, of finite volume, with both finite and ideal vertices, such that each facet of $\mathcal{R}_{n}$ is isometric to $\mathcal{R}_{n-1}$. Each $\mathcal{R}_{n}$ is produced by Whythoff's construction from the quotient of $\mathbb{H}^{n}$ by the reflective part of $O^{+}\left(f_{n}, \mathbb{Z}\right)$, with $f_{n}=-x_{0}^{2}+\sum_{k=1}^{n} x_{k}^{2}$, for $n=3, \ldots, 8$.

If we provide a non-orientable proper colouring of $\mathcal{R}_{3}$, as defined in [27, we can apply our previous reasoning in order to prove Proposition 1.2 and Theorem 1.4, as consequence. Let us label the faces of $\mathcal{R}_{3}$ as shown in Figure 3, and let the colouring be $\lambda=(1,1,4,7,5,2)$. It is easy to check that $\lambda$ is indeed proper, since we need to check only the colours around the finite vertices and edges of $\mathcal{R}_{3}$. Also, $\lambda$ is non-orientable, since $\lambda_{4}+\lambda_{5}+\lambda_{6}=\mathbf{0}$ in $\mathbb{Z}_{2}^{3}$. Let $\Gamma=\operatorname{ker} \lambda$.

Let $s_{i}$ be the reflection in the $i$-th facet of $\mathcal{R}_{3}$, and $\Gamma_{6}=\left\langle s_{1}, \ldots, s_{6}\right\rangle$, and $\Delta=\left\langle s_{1}, s_{2}, s_{3}\right\rangle$. Observe that $\Delta$ contains a free group of rank 2 as a normal subgroup of index 4 . Indeed, $F_{2}=\langle x, y\rangle$, with $x=s_{1} s_{2}, y=s_{3} s_{1} s_{2} s_{3}$ is such a subgroup.

Let $R$ be a retraction $\Gamma_{6} \rightarrow \Delta$ given by

$$
R: s_{i} \mapsto \begin{cases}s_{i}, & \text { if } i \in\{1,2,3\} \\ \text { id, } & \text { otherwise. }\end{cases}
$$

Since $R$ maps $x$ and $y$ respectively to themselves, and $\lambda\left(s_{1} s_{2}\right)=(1,0,0)^{t}+(1,0,0)^{t}=\mathbf{0}$ in $\mathbb{Z}_{2}^{3}$, we have that $F_{3} \subset R(\Gamma)$. Moreover, for $\delta=s_{4} s_{5} s_{6}$ it holds that $\lambda(\delta)=\mathbf{0}$, as already verified above, and $R(\delta)=$ id. Then the argument from the previous case of the right-angled dodecahedron applies verbatim.

For the induction step from $\mathcal{R}_{n-1}$ to $\mathcal{R}_{n}$ we just need to enhance the colouring in the way completely analogous to the extension of a non-orientable colouring of the dodecahedron $\mathcal{D}$ to a 
non-orientable colouring of the 120 -cell $\mathcal{C}$. Again, the rest of the argument proceeds verbatim in complete analogy to the previous cases.

2.4. Surfaces that bound geometrically. Let $\mathcal{P} \subset \mathbb{H}^{2}$ be a compact regular right-angled octagon, with sides labelled anti-clockwise $1,6,2,7,3,8,4,5$. Let $s_{i}$ be the reflection in the $i$-th side of $\mathcal{P}$, and $\Gamma_{8}=\operatorname{Ref}(\mathcal{P})=\left\langle s_{1}, s_{2}, \ldots, s_{8}\right\rangle$ be its reflection group. Also, let $\Gamma_{4}=\left\langle s_{1}, s_{2}, s_{3}, s_{4}\right\rangle$ and $F_{3}=\langle x, y, z\rangle$, with $x=s_{1} s_{2}, y=s_{1} s_{3}, z=s_{1} s_{4}$, be a free subgroup of $\Gamma_{4}$ of index 2 . The retraction of $\Gamma_{8}$ onto $\Gamma_{4}$ is given by

$$
R: s_{i} \mapsto \begin{cases}s_{i}, & \text { if } i \in\{1,2,3,4\}, \\ \text { id, } & \text { otherwise. }\end{cases}
$$

Let us choose a colouring $\lambda=(1,1,1,1,2,3,5,6)$ for $\mathcal{P}$, which is a proper and non-orientable one, since $\lambda_{1}+\lambda_{5}+\lambda_{6}=\mathbf{0} \in \mathbb{Z}_{2}^{3}$. Let $\Gamma=\operatorname{ker} \lambda$. An easy check ensures that $F_{3} \subset R(\Gamma)$, as well as that $R(\delta)=\mathrm{id}$ for an orientation-reversing element $\delta=s_{6} s_{7} s_{8} \in \Gamma$. Then the lower bound $\alpha(x) \geq(c x)^{\frac{x}{32 \pi}}$, for some constant $c>0$, for the number of geometrically bounding surfaces of area $\leq x$ (for $x$ large enough) follows immediately: the area of $\mathcal{P}$ equals $2 \pi, \Gamma$ has index 8 in $\Gamma_{8}$, and the orientation cover of a non-orientable surface has twice its area. We also use the fact that the rank $d \geq 2$ free group $F_{d}$ has $\geq(n !)^{d-1}$ subgroups of index $\leq n$, for $n$ large enough. The upper bound $\alpha(x) \leq(d x)^{\frac{x}{2 \pi}}$, for some constant $d \geq c>0$, follows from [4]. Since area $=4 \pi(g-1)$, for an orientable genus $g \geq 2$ surface, this proves Theorem 1.5. The case of non-compact finite-area surfaces mentioned in Remark 1.6 proceeds by analogy.

\section{Constructing Geodesic boundaries by arithmetic Reductions}

We start by recalling the following lemma of Long and Reid [30, Lemma 2.2] (c.f. also the remark after its proof).

Lemma 3.1 (Subgroup Lemma). Let $\Gamma<O^{+}(n, 1)$ be a subgroup of hyperbolic isometries defined over a number field $K$, and $\delta$ an element of $\Gamma$. Let $\theta_{1}, \theta_{2}: \Gamma \rightarrow F_{i}$ be two homomorphisms of $\Gamma$ onto a group $F_{i}$, with torsion-free kernels. Let $\Theta(g)=\left(\theta_{1}(g), \theta_{2}(g)\right): \Gamma \rightarrow F_{1} \times F_{2}$. Suppose that $\theta_{i}(\delta)$ has order $k_{i}<\infty, i=1,2$, and any prime dividing $\operatorname{gcd}\left(k_{1}, k_{2}\right)$ appears with distinct exponents in $k_{1}$ and $k_{2}$. Then $\Theta^{-1}\left\langle\left(\theta_{1}(\delta), \theta_{2}(\delta)\right)\right\rangle$ is a torsion-free subgroup in $\Gamma$ of finite index that contains $\delta$.

The following lemma is used in order to show that the maps that we choose in the sequel as $\theta_{i}$, $i=1,2$, in the Subgroup Lemma above have torsion-free kernels. Its proof is very similar to that of [30, Lemma 2.4].

Lemma 3.2 (No Torsion Lemma). Let $\Gamma<O^{+}(n, 1)$ be a finite subgroup defined over the ring of integers $\mathcal{O}_{K}$ of a number field $K$, and let $p \in \mathcal{O}_{K}$ be an odd rational prime that does not divide the order of $\Gamma$. Then the reduction of $\Gamma$ modulo the ideal $\mathcal{J}=(p)$ is isomorphic to $\Gamma$.

Proof. A non-trivial element $g$ of the kernel of the reduction map $\Gamma\left(\mathcal{O}_{K}\right) \rightarrow \Gamma\left(\mathcal{O}_{K} / \mathcal{J}\right)$ can be written in the form $g=\mathrm{id}+p^{r} h$, where $h$ is a matrix not all of whose entries are divisible by $p^{r}$, with $r$ some positive integer. Let $q<\infty$ be the order of an element $g \in \Gamma$. Then we get

$$
\mathrm{id}=g^{q}=\mathrm{id}+q p^{r} h+\sum_{t=2}^{q}\left(\begin{array}{l}
q \\
t
\end{array}\right) p^{r t} h^{t},
$$

and thus

$$
q h=\mathbf{0} \bmod p^{r} .
$$

The latter implies $p^{r}$ divides $q$, since $h \neq \mathbf{0} \bmod p^{r}$. Thus, $p$ divides $q$, and $q$ divides the order of $\Gamma$, since $q$ is the order of an element of $\Gamma$. The latter is a contradiction, and thus the reduction map has trivial kernel. 
As shown by Vinberg in [44, in some cases for an admissible quadratic form $q$ of signature $(n, 1)$ defined over a totally real number field $K$ with ring of integers $O_{K}$ it holds that $O^{+}\left(q, O_{K}\right)=$ $\operatorname{Ref}(P) \rtimes \operatorname{Sym}(P)$, where $P \subset \mathbb{H}^{n}$ is a finite-volume polytope. Here, $\operatorname{Ref}(P)$ denotes the associated reflection group, and $\operatorname{Sym}(P)$ is the group of symmetries of $P$. Also, we assume that $O_{K}$ is a principal ideal domain in order to keep our account simpler. We refer the reader to [17] for more details.

If the above presentation of $O^{+}\left(q, O_{K}\right)$ takes place for some finite-volume polytope $P \subset \mathbb{H}^{n}$, the form $q$ is called reflective, and the polytope $P$ is called its associated polytope.

An algorithm introduced by Vinberg in 44 and implemented in 18 by Guglielmetti allows us to find the associated polytope $P \subset \mathbb{H}^{n}$ in finite time, for any reflective admissible quadratic form of signature $(n, 1)$.

3.1. Compact polytopes in dimensions 5 and 6. Let $\omega=\frac{1+\sqrt{5}}{2}$ and let $P_{n} \subset \mathbb{H}^{n}$ be the polytopes associated to the quadratic forms

$$
\begin{gathered}
q_{5}=-(-1+2 \omega) x_{0}^{2}+x_{1}^{2}+x_{2}^{2}+x_{3}^{2}+x_{4}^{2}+x_{5}^{2}, \\
q_{6}=-2 \omega x_{0}^{2}+x_{1}^{2}+x_{2}^{2}+x_{3}^{2}+x_{4}^{2}+x_{5}^{2}+x_{6}^{2} .
\end{gathered}
$$

The polytopes $P_{5}$ and $P_{6}$ are apparently new, and were found by using AlVin and CoxIter software [18, 19]. They differ substantially from the polytopes that appear in [7, 8], and have fewer facets.

Let $\Gamma_{n}=\operatorname{Ref}\left(P_{n}\right)$, be the reflection group of $P_{n}$, with generators $s_{i}, i \in I_{n}$, where $I_{n}$ is the set of outer normals to the facets of $P_{n}$ or, equivalently, the set of nodes of the Coxeter diagram of $P_{n}^{1}$. With standard basis $\left\{v_{0}, v_{1}, \ldots, v_{n}\right\}$, the Vinberg algorithm determines the outer normals for $n=5,6$ which are given in the Appendix.

The associated reflection group $\Gamma_{n}$ is arithmetic, and contains a virtually free parabolic subgroup

$$
\Delta= \begin{cases}\left\langle s_{5}, s_{6}, s_{9}\right\rangle, & \text { for } n=5, \\ \left\langle s_{6}, s_{9}, s_{17}\right\rangle, & \text { for } n=6 ;\end{cases}
$$

Indeed, $\Delta$ is isomorphic to the $(2, \infty, \infty)$-triangle groun 2 , which contains $F_{2}$ as a subgroup of index 4.

The retraction $R: \Gamma_{n} \rightarrow \Delta$ is defined by sending all but three generators of $\Gamma_{n}$ to id, with the only generators mapped identically being those of $\Delta<\Gamma_{n}$.

In order for $R$ being well-defined, we essentially need that the generators of $\Delta$ be connected to the rest of the diagram by edges with even labels only, since any two generators connected by a path of odd-labelled edges are conjugate. This folds, for instance, if the facets corresponding to the reflections generating $\Delta$ are redoubleable in terms of [1].

The element

$$
\delta= \begin{cases}s_{1} s_{2} s_{3} s_{4} s_{7}, & \text { for } n=5, \\ s_{7} s_{13} s_{18}, & \text { for } n=6 .\end{cases}
$$

is orientation-reversing, as it is a product of an odd number of reflections in $\mathbb{H}^{n}$. Moreover, $\delta \in \operatorname{ker} R$.

We shall set the map $\Theta$ from Lemma 3.1 to be a pair of reductions modulo various rational primes, and then use Lemma 3.2 in order to ensure that their kernels are torsion-free. Indeed, we need to choose such an odd prime $p \in \mathbb{Z}$ that it does not divide the order of any finite parabolic

\footnotetext{
${ }^{1}$ The notation used is as follows: a dashed edge means two reflection hyperplanes have a common perpendicular, a solid edge means parallel (at the ideal boundary) hyperplanes, a double edge means label 4, a single edge means label 3 , any other edge has a label on it describing the corresponding dihedral angle. The colours are used for convenience only.

${ }^{2}$ Here $\Delta$ is not actually generated by reflections in the sides of a hyperbolic finite-area triangle, but is rather only abstractly isomorphic to such a group. However, we are interested in its algebraic rather than geometric properties, regarding its subgroup growth.
} 


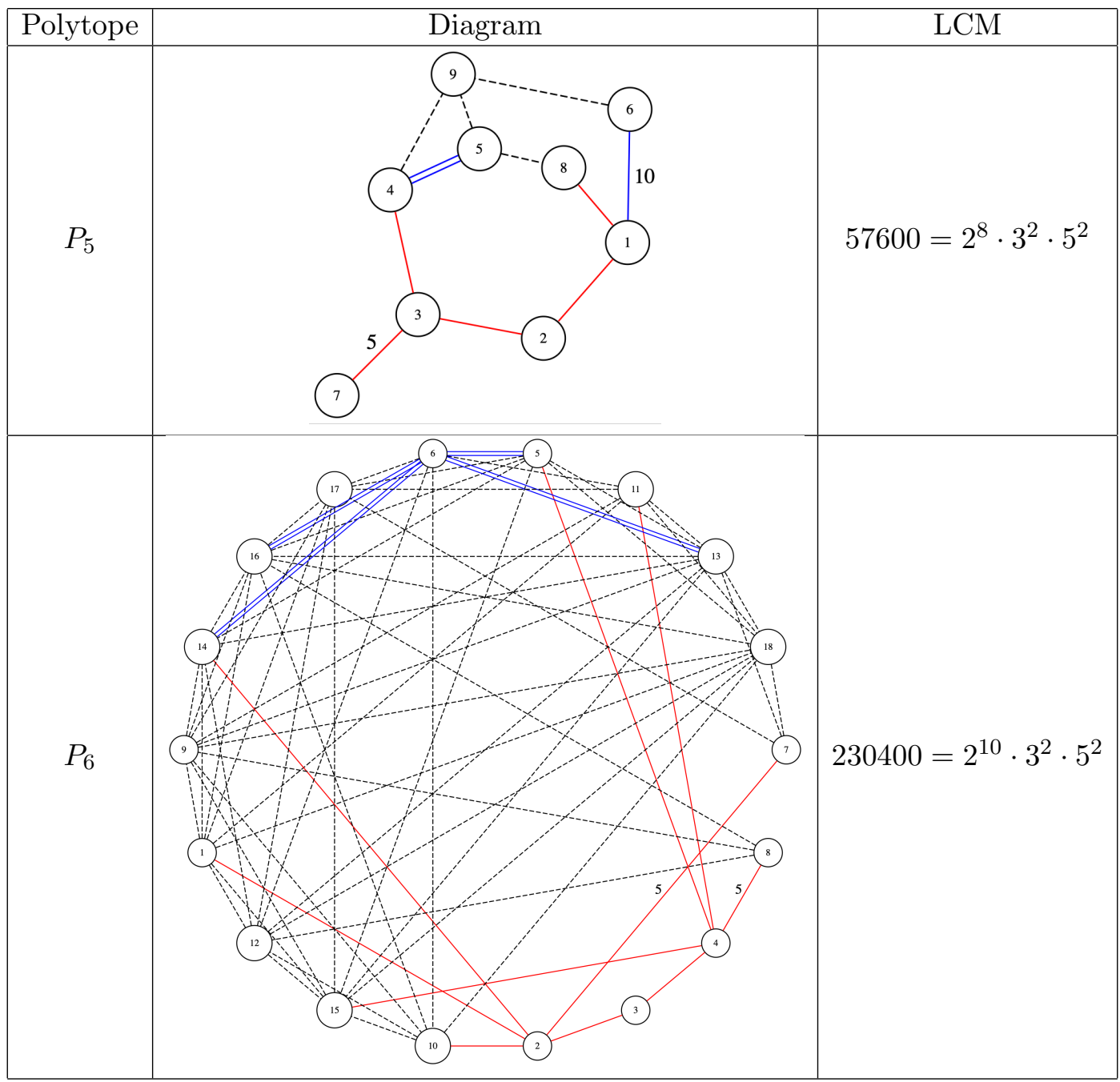

TABle 1. Polytopes $P_{n}, n=5,6$, their Coxeter diagrams, and the least common multiple (LCM) of the orders of their parabolic finite subgroups

subgroup in the diagram of $P_{n}, n=5,6$. The least common multiples of orders of finite parabolic subgroups for $P_{n}, n=5,6$, are given in Table 1 .

For $\Gamma<\mathrm{GL}\left(n+1, \mathcal{O}_{K}\right)$, where $\mathcal{O}_{K}$ is the ring of integers of a number field $K$, let $\phi_{p}$ denote the homomorphism $\Gamma \rightarrow \mathrm{GL}\left(n+1, \mathcal{O}_{K} / \mathcal{J}\right)$ induced by reduction modulo $\mathcal{J}=(p)$, the principal ideal generated by a rational integer $p$.

Let us consider the reductions $\phi_{7}$ and $\phi_{11}$ as defined above, and let $\Theta=\left(\phi_{7}, \phi_{11}\right)$. For $n=5$, the order of $\phi_{7}(\delta)$ equals $800=2^{5} \cdot 5^{2}$, while the order of $\phi_{11}(\delta)$ equals $8052=2^{2} \cdot 3^{1} \cdot 11^{1} \cdot 61^{1}$, as follows by straightforward computations, c.f. [10, 11]. For $n=6$, the order of $\phi_{7}(\delta)$ equals $8=2^{3}$, while the order of $\phi_{11}(\delta)$ equals $44=2^{2} \cdot 11^{1}$.

Then Lemma 3.1 and Lemma 3.2 apply, and $\Gamma=\Theta^{-1}\left\langle\left(\phi_{7}(\delta), \phi_{11}(\delta)\right)\right\rangle$ is a torsion-free subgroup of finite index in $\Gamma_{n}$ that contains the orientation-reversing element $\delta$ and retracts onto the free group $\Gamma \cap \Delta$. Then the argument analogous to that of Section 2 applies.

\footnotetext{
${ }^{3}$ The orders of all finite parabolic subgroups associated with $P_{n}$ can be obtained by using CoxIter [18] with the -debug option.
} 
3.2. Right-angled cusped polytopes in dimensions 4 to 8. Let $P_{n} \subset \mathbb{H}^{n}$ be the right-angled polytopes associated to the principal congruence subgroups of level 2 for the quadratic forms

$$
f_{n}=-x_{0}^{2}+x_{1}^{2}+x_{2}^{2}+\cdots+x_{n}^{2}, \text { for } n=4, \ldots, 8 .
$$

Let $\Gamma_{n}=\operatorname{Ref}\left(P_{n}\right)$, be the associated reflection group, with generators $s_{i}, i \in I_{n}$, where $I_{n}$ is the set of outer normals to the facets of $P_{n}$. With standard basis $\left\{v_{0}, v_{1}, \ldots, v_{n}\right\}$, the Vinberg algorithm starts with the first $n$ outer normals being

$$
e_{i}=-v_{i}, \text { for } 1 \leq i \leq n,
$$

and continues with the next $\left(\begin{array}{l}n \\ 2\end{array}\right)$ outer normals

$$
e_{i, j}=v_{0}+v_{i}+v_{j}, \text { for } 1 \leq i<j \leq n,
$$

all of them being 1-roots, as is necessary for determining the reflective part of the principle congruence level 2 subgroup, rather than that of the whole group of units for $f_{n}$. Let us set $e_{n+1}=e_{1,2}=v_{0}+v_{1}+v_{2}$ and $e_{n+2}=e_{3,4}=v_{0}+v_{3}+v_{4}$, as a more convenient notation.

Such $\Gamma_{n}$ is arithmetic, and it contains a virtually free parabolic subgroup $\Delta=\left\langle s_{3}, s_{4}, s_{n+2}\right\rangle$. Indeed, $\Delta$ is isomorphic to the $(2, \infty, \infty)$-triangle group which contains $F_{2}$ as a subgroup of index 4. Consider the retraction

$$
R: s_{i} \mapsto \begin{cases}s_{i}, & \text { if } i \in\{3,4, n+2\} \\ \text { id, } & \text { otherwise. }\end{cases}
$$

The element $\delta=s_{1} s_{2} s_{n+1}$ is orientation-reversing, as it is a product of 3 reflections in $\mathbb{H}^{n}$. Moreover, $\delta \in \operatorname{ker} R$.

For $\Gamma \in \operatorname{GL}(n+1, \mathbb{Z})$, let $\phi_{m}$ denote the homomorphism $\Gamma \rightarrow \operatorname{GL}(n+1, \mathbb{Z} / m \mathbb{Z})$ induced by reduction modulo a positive integer $m$. By [35, Theorem IX.7] we know that the kernel of $\phi_{m}$ is torsion-free for $m>2$. The reduction of $\delta$ modulo 3 has order $4=2^{2}$, while its reductions modulo 4 has order 2, c.f. [10, 11]. Letting $\Theta=\left(\phi_{3}, \phi_{4}\right)$, Lemma 3.1 applies, and $\Gamma=\Theta^{-1}\left\langle\left(\phi_{3}(\delta), \phi_{4}(\delta)\right)\right\rangle$ is a torsion-free subgroup of finite index in $\Gamma_{n}$ that contains the orientation-reversing element $\delta$ and retracts onto the free group $\Gamma \cap \Delta$.

3.3. Cusped polytopes in dimensions 9 to 13. Let $P_{n} \subset \mathbb{H}^{n}$ be the polytopes from Table 7 in [44] associated to the quadratic forms

$$
f_{n}=-2 x_{0}^{2}+x_{1}^{2}+x_{2}^{2}+\cdots+x_{n}^{2} \text { for } n=9,10 \text { and } 13,
$$

while $P_{n}$, for $n=11,12$, be the polytopes with Coxeter diagrams given in Figure 4, respectively. The latter ones appear to be new, and were found by using AlVin [19.

Let $\Gamma_{n}=\operatorname{Ref}\left(P_{n}\right)$, be the reflection group of $P_{n}$, with generators $s_{i}, i \in I_{n}$, where $I_{n}$ is the set of nodes in the Coxeter diagram of $P_{n}$. Such $\Gamma_{n}$ is arithmetic, and it contains a virtually free parabolic subgroup $\Delta$ indicated in Table 2 .

Since $\Delta$ is generated by reflections in redoubleable facets we can define a retraction $R: \Gamma_{n} \rightarrow \Delta$, as before, that send all the generators of $\Gamma_{n}$ to id, except of those of $\Delta$.

\begin{tabular}{|c|c|c|}
\hline$n$ & generators of $\Delta$ & triangle group $\cong \Delta$ \\
\hline 9 & $s_{9}, s_{10}, s_{12}$ & $(2, \infty, \infty)$ \\
10 & $s_{10}, s_{11}, s_{13}$ & $(2,4, \infty)$ \\
11 & $s_{11}, s_{12}, s_{18}$ & $(4,4, \infty)$ \\
12 & $s_{12}, s_{13}, s_{20}$ & $(4,4, \infty)$ \\
13 & $s_{13}, s_{14}, s_{19}$ & $(2, \infty, \infty)$ \\
\hline
\end{tabular}

TABLE 2. A virtually free parabolic subgroup $\Delta<\Gamma_{n}$, for $n=9, \ldots, 13$ 


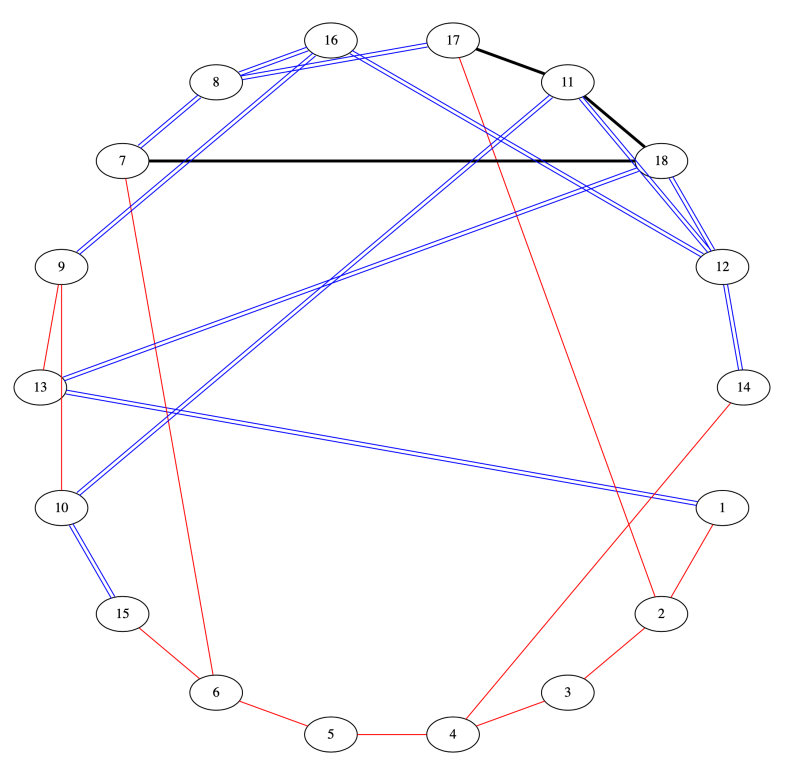

(a) $f_{11}=-2 x_{0}^{2}+x_{1}^{2}+\ldots+x_{8}^{2}+2 x_{9}^{2}+2 x_{10}^{2}+2 x_{11}^{2}$

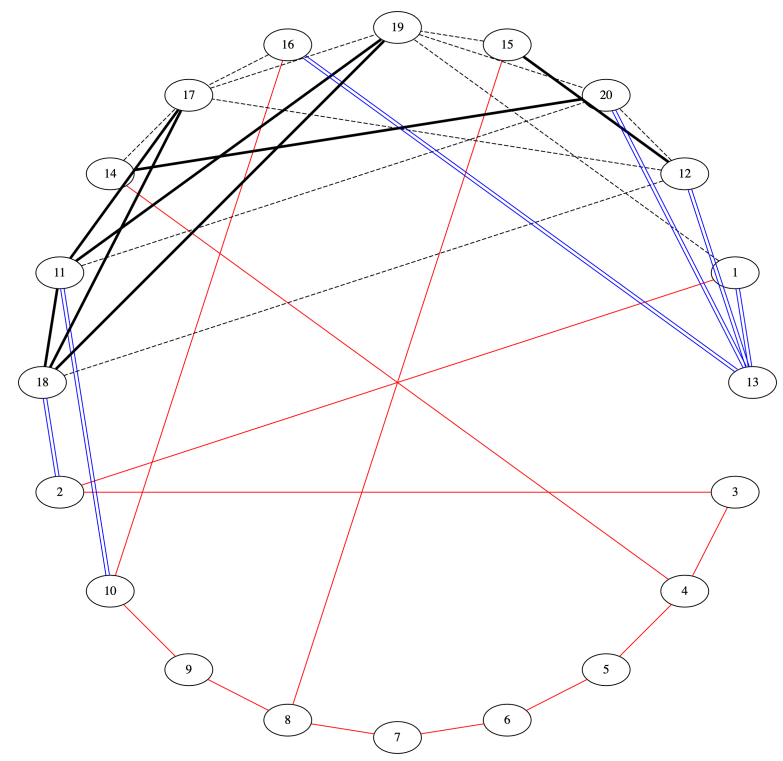

(b) $f_{12}=-2 x_{0}^{2}+x_{1}^{2}+\ldots+x_{11}^{2}+2 x_{12}^{2}$

Figure 4. The Coxeter diagrams for (a) $P_{11}$ and (b) $P_{12}$, together with their associated quadratic forms

The orientation-reversing element $\delta_{n} \in \operatorname{ker} R$ is defined by

$$
\delta_{n}= \begin{cases}s_{1} s_{2} s_{3} \ldots s_{8} s_{n+2}, & \text { for } n=9,10,13, \\ s_{7} s_{8} s_{16}, & \text { for } n=11, \\ s_{2} s_{11} s_{18}, & \text { for } n=12 .\end{cases}
$$

Letting $\Theta=\left(\phi_{m_{1}}, \phi_{m_{2}}\right)$, Lemma 3.1 applies with $m_{1}$ and $m_{2}$ as in Table 3. Here, we also notice that $\delta_{n}$ for $n=10,13$ is an extension of $\delta_{9}$ by the identity map, which simplifies the computations.

Then $\Gamma=\Theta^{-1}\left\langle\left(\phi_{m_{1}}(\delta), \phi_{m_{2}}(\delta)\right)\right\rangle$ is a torsion-free subgroup of finite index in $\Gamma_{n}$ that contains the orientation-reversing element $\delta$ and retracts onto the free group $\Gamma \cap \Delta$.

\begin{tabular}{|c|c|c|c|c|}
\hline$n$ & $m_{1}$ & $k_{1}=$ order of $\phi_{m_{1}}\left(\delta_{n}\right)$ & $m_{2}$ & $k_{2}=$ order of $\phi_{m_{2}}\left(\delta_{n}\right)$ \\
\hline $9,10,13$ & 3 & $84=2^{2} \cdot 3^{1} \cdot 7^{1}$ & 4 & $34=2^{1} \cdot 17^{1}$ \\
11,12 & 3 & $6=2^{1} \cdot 3^{1}$ & 4 & $4=2^{2}$ \\
\hline
\end{tabular}

TABle 3. Orders of the reductions of $\delta$ and their prime factorisations, c.f. [10, 11]

\section{Constructing geometric boundaries from virtual Retracts}

Let $q_{n}$ be an admissible quadratic form of signature $(n, 1)$ defined over a totally real number field $K$ with ring of integers $O_{K}$, and let $q_{n+1}=q_{n}+x_{n+1}^{2}$. Suppose also that $\Gamma_{n}<O^{+}\left(q_{n}, O_{K}\right)$ is a torsion-free subgroup, either of finite co-volume or co-compact.

Now assume that there exists a retraction $R_{n}: \Gamma_{n} \rightarrow \Delta$ of $\Gamma_{n}$ onto a virtually free subgroup $\Delta$ such that ker $R_{n}$ contains an orientation-reversing element $\delta$.

By [24, Proposition 7.1], there exists a torsion-free finite-index subgroup $\Gamma_{n+1}^{\prime} \leq O^{+}\left(q_{n+1}, O_{K}\right)$, such that $\Gamma_{n}<\Gamma_{n+1}^{\prime}$. Moreover, we may assume that $\mathbb{H}^{n} / \Gamma_{n}$ is a properly embedded totally geodesic submanifold of $\mathbb{H}^{n+1} / \Gamma_{n+1}$. Thus, the group $\Gamma_{n}$ is a geometrically finite subgroup of $\Gamma_{n+1}^{\prime}$. By [5, Theorem 1.4], there is a finite index subgroup $G<\Gamma_{n+1}^{\prime}$, such that $G$ virtually retracts to its 
geometrically finite subgroups. In particular, $G$ virtually retracts to $G \cap \Gamma_{n}$. However, since $\Gamma_{n+1}^{\prime}$ is linear, the arguments of [31, Theorem 2.10] apply to give a virtual retraction from $\Gamma_{n+1}^{\prime}$ onto $\Gamma_{n}$. Let $\Gamma_{n+1}$ be the finite index subgroup of $\Gamma_{n+1}^{\prime}$ which retracts onto $\Gamma_{n}$. Then the composition $R_{n+1}: \Gamma_{n+1} \rightarrow \Gamma_{n} \rightarrow \Delta$ is a retraction of $\Gamma_{n+1}$ onto $\Delta$, such that $\delta \in \operatorname{ker} R_{n+1}$.

All the previous arguments from Section 3 apply, and we obtain Theorems $1.4-1.5$ for all $n \geq 2$, since we can use any of our examples worked out in Sections 23 as a basis for the above inductive procedure.

\section{ACKNowledgements}

The first author thanks Darren Long for many helpful discussions. Both authors would like to thank Mikhail Belolipetsky (IMPA), Vincent Emery (Universität Bern), and Ruth Kellerhals (Université de Fribourg), as well as the Mathematisches Forschungsinstitut Oberwolfach (MFO) administration, for organising the miniworkshop "Reflection Groups in Negative Curvature" (1915b) in April 2019, during which the results of this paper were presented and discussed. They also thank the organisers of the Borel Seminar (Les Diablerets, Switzerland) in December 2018, where this work was started, for stimulating research atmosphere, and the Swiss Doctoral Program - CUSO, Swiss Mathematical Society, and ETH Zürich for the financial support of the event. A word of special gratitude is addressed to the creators and maintainers of SAGE Math, and to Rafael Guglielmetti, the author of CoxIter and AlVin. The aforementioned software made most of the calculations in the present work possible and provided means for additional verification of those previously

done. We also thank the anonymous referees for their suggestions and criticism that helped us improving this paper.

\section{REFERENCES}

[1] D. Allcock, Infinitely many hyperbolic Coxeter groups through dimension 19, Geom. Topol. 10 (2006), 737-758.

[2] E. M. Andreev, On convex polyhedra in Lobačevskiu space, Math. USSR Sb. 10 (1970), 413-440.

[3] M. Belolipetsky, Arithmetic hyperbolic reflection groups, Bulletin AMS 53 (2016), 437-475.

[4] M. Belolipetsky - T. Gelander - A. Lubotzky - A. Shalev, Counting arithmetic lattices and surfaces, Ann. of Math. (2) $\mathbf{1 7 2}$ (2010), 2197-2221.

[5] N. Bergeron - F. Haglund - D. T. Wise, Hyperplane sections in arithmetic hyperbolic manifolds, J. London Math. Soc. 83 (2011), 431-448.

[6] R. Brooks, Circle packings and co-compact extensions of Kleinian groups, Invent. Math. 86 (1986), 461-469.

[7] V. O. Bugaenko, Groups of automorphisms of unimodular hyperbolic quadratic forms over the ring $\mathbb{Z}\left[\frac{1+\sqrt{5}}{2}\right]$, Vestnik Moskov. Univ. Ser. I Mat. Mekh., 5 (1984), 6-12.

[8] __ Arithmetic crystallographic groups generated by reflections, and reflective hyperbolic lattices, Lie groups, their discrete subgroups, and invariant theory, 33-55, Adv. Soviet Math., 8, Amer. Math. Soc., Providence, RI, 1992.

[9] M. Burger - T. Gelander - A. Lubotzky - S. Mozes: Counting hyperbolic manifolds, Geom. Funct. Anal. 12 (2002), 1161-1173.

[10] M. Chu, A. KolPaKov, Auxiliary Wolfram Mathematica notebooks, https://doi.org/10.7910/DVN/NODP7R

[11] M. Chu, A. KolPakov, Auxiliary SageMath worksheets, https://doi.org/10.7910/DVN/NODP7R

[12] M. Davis - T. Januszkiewicz, Convex polytopes, Coxeter orbifolds and torus actions, Duke Math. J. 62 (1991), 417-451.

[13] F. T. FArrell - S. Zdravkovska, Do almost flat manifolds bound?, Michigan Math. J. 30 (1983), $199-208$.

[14] A. Garrison - R. Scott, Small covers of the dodecahedron and the 120-cell, Proc. Amer. Math. Soc. 131 (2003), 963-971.

[15] M. Gromov, Manifolds of negative curvature, J. Differential Geom. 13 (1987), 223-230.

[16] _ Almost flat manifolds, J. Differential Geom. 13 (1978), 231-241.

[17] R. Guglielmetti, Hyperbolic isometries in (in-)finite dimensions and discrete reflection groups: theory and computations, Ph.D. thesis no. 2008, Université de Fribourg (2017).

[18] _ CoxIter - Computing invariants of hyperbolic Coxeter groups, LMS Journal of Computation and Mathematics 18 (2015), 754-773.

[19] - AlVin: a C++ implementation of the Vinberg algorithm for diagonal quadratic forms, https://rgugliel.github.io/AlVin/ 
[20] I. V. Izmestiev, Three-dimensional manifolds defined by coloring a simple polytope, Math. Notes 69 (2001), $340-346$.

[21] I. M. KaplinskayA - 亡̀. B. Vinberg, On the groups $O_{18,1}(\mathbb{Z})$ and $O_{19,1}(\mathbb{Z})$, Dokl. Akad. Nauk SSSR 238 (1978), 1273-1275.

[22] D. A. Kazhdan - G. A. Margulis, A proof of Selberg's hypothesis, Math. Sbornik 75 (1968), $162-168$.

[23] A. Kolpakov - B. Martelli - S. T. Tschantz, Some hyperbolic three-manifolds that bound geometrically, Proc. AMS 143 (2015), 4103-4111.

[24] A. Kolpakov - A. Reid - L. Slavich, Embedding arithmetic hyperbolic manifolds, Math. Res. Lett. 25 (2018), 1305-1328.

[25] A. Kolpakov - A. Reid - S. Riolo, Many cusped hyperbolic 3-manifolds do not bound geometrically, Proc. Amer. Math. Soc. 148 (2020), 2233-2243.

[26] A. Kolpakov - S. Riolo, Counting cusped hyperbolic three-manifolds that bound geometrically, Trans. Amer. Math. Soc. 373 (2020), 229-247.

[27] A. Kolpakov - L. Slavich, Hyperbolic 4-manifolds, colourings and mutations, Proc. LMS 113 (2016), $163-184$.

[28] D. D. Long - A. W. REID, On the geometric boundaries of hyperbolic 4-manifolds, Geom. Topol. 4 (2000), 171-178.

[29] _ All flat manifolds are cusps of hyperbolic orbifolds, Alg. Geom. Topol. 2 (2002), 285-296.

[30] _ Constructing hyperbolic manifolds which bound geometrically, Math. Research Lett. 8 (2001), $443-456$.

[31] _ Subgroup separability and virtual retractions of groups, Topology 47 (2008), 137-159.

[32] A. LubotzKY, Subgroup growth and congruence subgroups, Invent. Math. 119 (1995), 267-295.

[33] J. MA, F. Zheng, Geometrically bounding 3-manifolds, volume and Betti numbers, arXiv:1704.02889.

[34] C. Maclachlan - A. W. Reid, The arithmetic of hyperbolic 3-manifolds, Graduate Texts in Mathematics 219, Springer-Verlag, New York (2003).

[35] M. Newman, Integral matrices, Pure and Applied Mathematics 45, Academic Press: New York (1972).

[36] B. E. Nimershiem, All flat three-manifolds appear as cusps of hyperbolic four-manifolds, Topology and its Appl. 90 (1998), 109-133.

[37] L. Potyaga ̌llo - È. Vinberg, On right-angled reflection groups in hyperbolic spaces, Comment. Math. Helv. 80 (2005), 63-73.

[38] L. Slavich, A geometrically bounding hyperbolic link complement, Alg. Geom. Topol. 15 (2015), 1175-1197.

[39] _ The complement of the figure-eight knot geometrically bounds, Proc. AMS 145 (2017), 1275-1285.

[40] J. G. Ratcliffe - S. T. Tschantz, Gravitational instantons of constant curvature, Class. Quantum Grav. 15 (1998), 2613-2627.

[41] - On the growth of the number of hyperbolic gravitational instantons with respect to volume, Class. Quantum Grav. 17 (2000), 2999-3007.

[42] A. Yu. Vesnin, Three-dimensional hyperbolic manifolds of Löbell type, Siberian Math. J. 28 (1987), 731-733.

[43] _ Three-dimensional hyperbolic manifolds with a common fundamental polyhedron, Math. Notes 49 (1991), $575-577$.

[44] È. B. VinberG, On groups of unit elements of certain quadratic forms, Math. USSR - Sbornik 16 (1972), 17-35.

[45] È. B. Vinberg - O. V. Shvartsman, Discrete groups of motions of spaces of constant curvature, in: Geometry II, Enc. Math. Sci. 29, Berlin: Springer (1993), 139-248.

[46] H. C. WANG, Topics on totally discontinuous groups. Symmetric spaces, in: Short Courses, Washington University 8, New York: M. Dekker (1972), 459-487.

[47] B. Zimmermann, Hurwitz groups and finite group actions on hyperbolic 3-manifolds, Journal LMS 52 (1995), 199-208.

[48] _ A note on surfaces bounding hyperbolic 3-manifolds, Monats. Math. 142 (2004), 267-273. 


\section{Appendix A.}

A.1. Outer normals for compact $P_{5}$. Section 3.1

$e_{i}=-v_{i}+v_{i+1}$ for $1 \leq i \leq 4$,

$e_{5}=-v_{5}$,

$e_{6}=\omega v_{0}+(2+\omega) v_{1}$,

$e_{7}=\omega\left(v_{0}+v_{1}+v_{2}+v_{3}\right)$,

$e_{8}=(1+\omega)\left(v_{0}+v_{1}\right)+\omega\left(v_{2}+v_{3}+v_{4}+v_{5}\right)$.

A.2. Outer normals for compact $P_{6}$. Section 3.1

$e_{i}=-v_{i}+v_{i+1}$ for $1 \leq i \leq 5$,

$e_{6}=-v_{6}$,

$e_{7}=v_{0}+w\left(v_{1}+v_{2}\right)$,

$e_{8}=\omega\left(v_{0}+v_{1}+v_{2}+v_{3}+v_{4}\right)$,

$e_{9}=\omega v_{0}+2 \omega v_{1}$

$e_{10}=(1+\omega)\left(v_{0}+v_{1}+v_{2}\right)+\omega\left(v_{3}+v_{4}+v_{5}+v_{6}\right)$,

$e_{11}=(1+2 \omega) v_{0}+(1+3 \omega) v_{1}+(1+\omega)\left(v_{2}+v_{3}+v_{4}\right)+\omega\left(v_{5}+v_{6}\right)$,

$e_{12}=(1+2 \omega) v_{0}+(2+3 \omega) v_{1}+\omega\left(v_{2}+v_{3}+v_{4}+v_{5}+v_{6}\right)$,

$e_{13}=(2+2 \omega) v_{0}+(1+2 \omega)\left(v_{1}+v_{2}+v_{3}+v_{4}+v_{5}\right)+v_{6}$,

$e_{14}=(2+3 \omega) v_{0}+(2+4 \omega) v_{1}+(2+2 \omega) v_{2}+(1+2 \omega)\left(v_{3}+v_{4}+v_{5}\right)+v_{6}$,

$e_{15}=(2+3 \omega) v_{0}+(3+4 \omega) v_{1}+(1+2 \omega)\left(v_{2}+v_{3}+v_{4}\right)+2 \omega v_{5}$,

$e_{16}=(2+4 \omega) v_{0}+(3+6 \omega) v_{1}+(1+2 \omega)\left(v_{2}+v_{3}+v_{4}+v_{5}\right)+v_{6}$,

$e_{17}=(3+4 \omega) v_{0}+(2+5 \omega) v_{1}+(2+3 \omega)\left(v_{2}+v_{3}+v_{4}+v_{5}\right)+\omega v_{6}$,

$e_{18}=(4+5 \omega) v_{0}+(4+6 \omega) v_{1}+(2+4 \omega)\left(v_{2}+v_{3}+v_{4}+v_{5}\right)$.

A.3. Outer normals for cusped $P_{4}$. Section 3.2)

$e_{i}=-v_{i}$ for $1 \leq i \leq 4$,

$e_{i}=v_{0}+v_{j_{1}}+v_{j_{2}}$ for $5 \leq i \leq 10$ and $1 \leq j_{1}<j_{2} \leq 4$.

A.4. Outer normals for cusped $P_{5}$. Section 3.2

$e_{i}=-v_{i}$ for $1 \leq i \leq 5$,

$e_{i}=v_{0}+v_{j_{1}}+v_{j_{2}}$ for $6 \leq i \leq 15$ and $1 \leq j_{1}<j_{2} \leq 5$,

$e_{16}=2 v_{0}+v_{1}+v_{2}+v_{3}+v_{4}+v_{5}$.

A.5. Outer normals for cusped $P_{6}$. Section 3.2

$e_{i}=-v_{i}$ for $1 \leq i \leq 6$,

$e_{i}=v_{0}+v_{j_{1}}+v_{j_{2}}$ for $7 \leq i \leq 21$ and $1 \leq j_{1}<j_{2} \leq 6$,

$e_{i}=2 v_{0}+v_{j_{1}}+\cdots+v_{j_{5}}$ for $22 \leq i \leq 27$ and $1 \leq j_{1}<j_{2}<j_{3}<j_{4}<j_{5} \leq 6$.

A.6. Outer normals for cusped $P_{7}$. Section 3.2

$e_{i}=-v_{i}$ for $1 \leq i \leq 7$,

$e_{i}=v_{0}+v_{j_{1}}+v_{j_{2}}$ for $8 \leq i \leq 28$ and $1 \leq j_{1}<j_{2} \leq 7$,

$e_{i}=2 v_{0}+v_{j_{1}}+\cdots+v_{j_{5}}$ for $29 \leq i \leq 49$ and $1 \leq j_{1}<j_{2}<j_{3}<j_{4}<j_{5} \leq 7$,

$e_{i}=3 v_{0}+2 v_{j_{1}}+v_{j_{2}}+\cdots+v_{j_{7}}$ for $50 \leq i \leq 56$ and $1 \leq j_{1}<j_{2}<j_{3}<j_{4}<j_{5}<j_{6}<j_{7} \leq 7$. 
A.7. Outer normals for cusped $P_{8}$. (Section 3.2)

$e_{i}=-v_{i}$ for $1 \leq i \leq 8$,

$e_{i}=v_{0}+v_{j_{1}}+v_{j_{2}}$ for $9 \leq i \leq 36$ and $1 \leq j_{1}<j_{2} \leq 8$,

$e_{i}=2 v_{0}+v_{j_{1}}+\cdots+v_{j_{5}}$ for $37 \leq i \leq 92$ and $1 \leq j_{1}<j_{2}<j_{3}<j_{4}<j_{5} \leq 8$,

$e_{i}=3 v_{0}+2 v_{j_{1}}+v_{j_{2}}+\cdots+v_{j_{7}}$ for $93 \leq i \leq 148$,

$e_{i}=4 v_{0}+2\left(v_{j_{1}}+\cdots+v_{j_{3}}\right)+v_{j_{4}}+\cdots+v_{j_{7}}$ for $149 \leq i \leq 204$

and $1 \leq j_{1}<j_{2}<j_{3}<j_{4}<j_{5}<j_{6}<j_{7} \leq 7$,

$e_{i}=5 v_{0}+2\left(v_{j_{1}}+\cdots+v_{j_{6}}\right)+v_{j_{7}}+v_{j_{8}}$ for $205 \leq i \leq 232$,

$e_{i}=6 v_{0}+3 v_{j_{1}}+v_{j_{2}}+\cdots+v_{j_{8}}$ for $233 \leq i \leq 240$

and $1 \leq j_{1}<j_{2}<j_{3}<j_{4}<j_{5}<j_{6}<j_{7}<j_{8} \leq 8$.

A.8. Outer normals for cusped $P_{9}$. (Section 3.3)

$e_{i}=-v_{i}+v_{i+1}$, for $1 \leq i \leq 8$,

$e_{9}=-v_{9}$,

$e_{10}=v_{0}+2 v_{1}$,

$e_{11}=v_{0}+v_{1}+v_{2}+v_{3}+v_{4}$,

$e_{12}=2 v_{0}+v_{1}+v_{2}+v_{3}+v_{4}+v_{5}+v_{6}+v_{7}+v_{8}+v_{9}$.

A.9. Outer normals for cusped $P_{10}$. Section 3.3)

$e_{i}=-v_{i}+v_{i+1}$, for $1 \leq i \leq 9$,

$e_{10}=-v_{10}$,

$e_{11}=v_{0}+v_{1}+v_{2}+v_{3}+v_{4}$,

$e_{12}=v_{0}+2 v_{1}$,

$e_{13}=2 v_{0}+v_{1}+v_{2}+v_{3}+v_{4}+v_{5}+v_{6}+v_{7}+v_{8}+v_{9}+v_{10}$.

A.10. Outer normals for cusped $P_{11}$. Section 3.3

$e_{i}=-v_{i}+v_{i+1}$, for $1 \leq i \leq 7$, and $i=9,10$,

$e_{i}=-v_{i}$, and $i=8,11$,

$e_{12}=v_{0}+v_{9}+v_{10}+v_{11}$,

$e_{13}=v_{0}+2 v_{1}+v_{9}$,

$e_{14}=v_{0}+v_{1}+v_{2}+v_{3}+v_{4}$,

$e_{15}=2 v_{0}+v_{1}+v_{2}+v_{3}+v_{4}+v_{5}+v_{6}+v_{9}+v_{10}$,

$e_{16}=2 v_{0}+v_{1}+v_{2}+v_{3}+v_{4}+v_{5}+v_{6}+v_{7}+v_{8}+v_{9}$,

$e_{17}=3 v_{0}+2\left(v_{1}+v_{2}\right)+v_{3}+v_{4}+v_{5}+v_{6}+v_{7}+v_{8}+v_{9}+v_{10}+v_{11}$,

$e_{18}=4 v_{0}+2\left(v_{1}+v_{2}+v_{3}+v_{4}+v_{5}+v_{6}+v_{7}\right)+v_{9}+v_{10}+v_{11}$.

A.11. Outer normals for cusped $P_{12}$. (Section 3.3)

$e_{i}=-v_{i}+v_{i+1}$, for $1 \leq i \leq 10$,

$e_{i}=-v_{i}$, for $i=11,12$,

$e_{13}=v_{0}+2 v_{1}+v_{12}$,

$e_{14}=v_{0}+v_{1}+v_{2}+v_{3}+v_{4}$,

$e_{15}=2 v_{0}+v_{1}+v_{2}+v_{3}+v_{4}+v_{5}+v_{6}+v_{7}+v_{8}+v_{12}$,

$e_{16}=2 v_{0}+v_{1}+v_{2}+v_{3}+v_{4}+v_{5}+v_{6}+v_{7}+v_{8}+v_{9}+v_{10}$,

$e_{17}=3 v_{0}+v_{1}+v_{2}+v_{3}+v_{4}+v_{5}+v_{6}+v_{7}+v_{8}+v_{9}+v_{10}+v_{11}+2 v_{12}$, 
$e_{18}=3 v_{0}+2\left(v_{1}+v_{2}\right)+v_{3}+v_{4}+v_{5}+v_{6}+v_{7}+v_{8}+v_{9}+v_{10}+v_{11}+v_{12}$,
$e_{19}=3\left(v_{0}+v_{1}\right)+v_{2}+v_{3}+v_{4}+v_{5}+v_{6}+v_{7}+v_{8}+v_{9}+v_{10}+v_{11}$,
$e_{20}=5 v_{0}+2\left(v_{1}+v_{2}+v_{3}+v_{4}+v_{5}+v_{6}+v_{7}+v_{8}+v_{9}+v_{10}+v_{11}+v_{12}\right)$.

A.12. Outer normals for cusped $P_{13}$. Section 3.3)

$e_{i}=-v_{i}+v_{i+1}$, for $1 \leq i \leq 12$,

$e_{13}=-v_{13}$,

$e_{14}=v_{0}+v_{1}+v_{2}+v_{3}+v_{4}$,

$e_{15}=v_{0}+2 v_{1}$,

$e_{16}=2 v_{0}+v_{1}+v_{2}+v_{3}+v_{4}+v_{5}+v_{6}+v_{7}+v_{8}+v_{9}+v_{10}$,

$e_{17}=3 v_{0}+3 v_{1}+v_{2}+v_{3}+v_{4}+v_{5}+v_{6}+v_{7}+v_{8}+v_{9}+v_{10}+v_{11}+v_{12}$,

$e_{18}=3 v_{0}+2 v_{1}+2 v_{2}+v_{3}+v_{4}+v_{5}+v_{6}+v_{7}+v_{8}+v_{9}+v_{10}+v_{11}+v_{12}+v_{13}$,

$e_{19}=5 v_{0}+2\left(v_{1}+v_{2}+v_{3}+v_{4}+v_{5}+v_{6}+v_{7}+v_{8}+v_{9}+v_{10}+v_{11}+v_{12}+v_{13}\right)$.

Department of Mathematics, University of Illinois at Chicago, 851 S. Morgan Street, Chicago, IL 60607-7045, USA

E-mail address: michu@uic.edu

Institut de mathématiques, Université de Neuchâtel, Rue Emile-Argand 11, CH-2000 Neuchâtel, Suisse / SWitzerland

E-mail address: kolpakov.alexander@gmail.com 